Robin de Jong

\title{
Local invariants attached to Weierstrass points
}

Received: 23 June 2008 / Revised: 8 December 2008

Published online: 17 March 2009

(C) The Author(s) 2009. This article is published with open access at Springerlink.com

\begin{abstract}
Let $X / S$ be a hyperelliptic curve of genus $g$ over the spectrum of a discrete valuation ring. Two fundamental numerical invariants are attached to $X / S$ : the valuation $d$ of the hyperelliptic discriminant of $X / S$, and the valuation $\delta$ of the Mumford discriminant of $X / S$ (equivalently, the Artin conductor). For a residue field of characteristic 0 as well as for $X / S$ semistable the invariants $d$ and $\delta$ are known to satisfy certain inequalities. We prove an exact formula relating $d$ and $\delta$ with intersection theoretic data determined by the distribution of Weierstrass points over the special fiber, in the semistable case. We also prove an exact formula for the stable Faltings height of an arbitrary curve over a number field, involving local contributions associated to its Weierstrass points.
\end{abstract}

\section{Introduction}

Let $R$ be a discrete valuation ring with perfect residue field $k$. Let $C$ be a smooth proper geometrically connected curve of genus $g \geq 1$ over $K=\operatorname{Fr}(R)$ and let $\rho: X \rightarrow S$ be the minimal regular model of $C$ over $S=\operatorname{Spec} R$. Let $\omega_{X / S}$ be the relative dualising sheaf of $X / S$. The Mumford isomorphism [25, Theorem 5.10]:

$$
\operatorname{det} R \rho_{*}\left(\omega_{X / S}^{\otimes 2}\right) \otimes K \stackrel{\sim}{\longrightarrow}\left(\operatorname{det} R \rho_{*} \omega_{X / S}\right)^{\otimes 13} \otimes K,
$$

which is well-defined up to a sign, gives a canonical rational section $\Delta$ of:

$$
\left(\operatorname{det} R \rho_{*} \omega_{X / S}\right)^{\otimes 13} \otimes \operatorname{det} R \rho_{*}\left(\omega_{X / S}^{\otimes 2}\right)^{\otimes-1},
$$

and a basic numerical invariant attached to $X / S$, namely the valuation $\delta_{X / S}=$ $\operatorname{ord}_{v}(\Delta)$ of $\Delta$. Here and in the rest of this paper we fix the convention that the valuation of a discrete valuation ring is normalised in the sense that its value group is $\mathbb{Z}$. It turns out that $\delta_{X / S}$ is non-negative and that actually $\delta_{X / S}=-\operatorname{Art}_{X / S}$, where $\operatorname{Art}_{X / S}$ is the Artin conductor of $X / S$ [29, Theorem 1]. If $X / S$ is semistable one can view $\Delta$ as the pullback, under the period map $S \rightarrow \overline{\mathcal{M}_{g}}$, of the tautological section corresponding to the boundary divisor of the algebraic stack $\overline{\mathcal{M}_{g}}$ of stable curves of genus $g$. In particular if $X / S$ is semistable $\delta_{X / S}$ is equal to the number of singular points in the geometric special fiber of $X / S$.

R. de Jong: Mathematical Institute, University of Leiden, PO Box 9512, 2300 RA Leiden, The Netherlands. e-mail: rdejong@math.leidenuniv.nl

Mathematics Subject Classification (2000): 14H55, 14H10, 14G40 
If $C$ is elliptic or hyperelliptic, one has a second basic invariant attached to $X / S$, namely $d_{X / S}=\operatorname{ord}_{v}(\Lambda)$ where $\Lambda$ in $H^{0}\left(S,\left(\operatorname{det} R \rho_{*} \omega_{X / S}\right)^{\otimes 8 g+4}\right)$ is the hyperelliptic discriminant associated to a hyperelliptic equation for $C / K$ (cf. end of section 3.1). Both $\delta_{X / S}$ and $d_{X / S}$ are zero if $X / S$ is smooth. For $C$ elliptic a result of Tate-Ogg [28] states the equality $d_{X / S}=\delta_{X / S}$ of both invariants. It follows from examples in [27] that in a number of cases with $g=2$ and with $k$ not of characteristic 2,3 or 5 , one has an equality $d_{X / S}=2 \delta_{X / S}$. However, as is shown in [33] there are exceptions to this rule, indicating that one should add a non-negative 'error term' $\varepsilon$ so that the formula $d_{X / S}=2 \delta_{X / S}+\varepsilon$ is true in general. Ueno in fact compiled a table containing values of $d_{X / S}$ and $\delta_{X / S}$ for $k$ not of characteristic 2, 3 or 5 based on the complete classification of degenerate fiber types [26] in genus two, showing precisely what the exceptions are. A more conceptual approach to the comparison of $d_{X / S}$ and $\delta_{X / S}$ in genus two was given subsequently by Saito [30] and Liu [18]. We state the result of Liu. Assume that $k$ is algebraically closed, and let $\pi: X \rightarrow X^{\prime}$ be the contraction of those irreducible components $\Gamma$ of the special fiber of $X / S$ for which $\left.\operatorname{deg} \omega_{X / S}\right|_{\Gamma}=0$. The hyperelliptic involution $\sigma$ of $X / S$ extends uniquely as an automorphism of $X^{\prime}$ over $S$. Let $Z=X^{\prime} /\langle\sigma\rangle$; this is a normal surface over $S$, with generic fiber isomorphic to $\mathbb{P}_{K}^{1}$. The following then holds: let $\tilde{Z} \rightarrow Z$ be the minimal desingularisation of $Z$. Let $n$ be the number of irreducible components of the special fiber of $\tilde{Z}$. Then $n$ is odd and:

$$
d_{X / S}=2 \delta_{X / S}+n-1 .
$$

For arbitrary hyperelliptic $C$, Matsusaka [20, Theorem 4.0.4] has shown the inequalities:

$$
g \delta_{X / S} \leq d_{X / S} \leq g^{2} \delta_{X / S}
$$

for $g$ even and:

$$
g \delta_{X / S} \leq d_{X / S} \leq\left(g^{2}+1\right) \delta_{X / S}
$$

for $g$ odd, in the case where $k$ has characteristic 0 . In particular $d_{X / S}$ is non-negative. These inequalities still hold for arbitrary $k$, provided one restricts to semistable hyperelliptic curves (for definitions see Sect. 3.1). In this case the inequalities follow from a well-known identity due, in increasing order of generality, to CornalbaHarris [5], Kausz [14], Maugeais [21] and Yamaki [35].

Our aim in this paper is to prove an exact formula relating $d_{X / S}$ and $\delta_{X / S}$ in arbitrary genus to intersection theoretic data associated to the distribution of Weierstrass points over the special fiber of $X / S$. At present we only have a result for semistable hyperelliptic curves, a circumstance due the point that we have to assume that all Weierstrass points on the generic fiber are rational. We hope that this restriction is in the end only of a technical nature and can be circumvented by methods as in e.g. [34, Sect. 3].

Here is our set-up. Let $F$ be the closure in $X$ of the Weierstrass points on $C$. For $P$ a section of $X / S$ we let $\Phi_{P}$ be the vertical $\mathbb{Q}$-Cartier divisor on $X$ uniquely determined by the following conditions: the divisor $(2 g-2) P-\omega_{X / S}+\Phi_{P}$ has intersection number 0 with each component $\Gamma$ of the geometric special fiber of 
$X / S$, and $P^{*} \Phi_{P}$ is the trivial divisor on $S$. It is known (cf. [19, Section 9.1.3]) that one has an intersection product $\operatorname{Div}_{S} X \times \operatorname{Div} X \rightarrow \mathbb{Z}$ where $\operatorname{Div} X$ is the group of Cartier divisors on $X$, and $\operatorname{Div}_{S} X \subset \operatorname{Div} X$ is the subgroup of Cartier divisors with support in the special fiber. In particular, one has the intersection number $\left(D, \omega_{X / S}\right)$ for $D$ supported in the special fiber. Also, one has the self-intersection $\Phi_{P}^{2}$ of $\Phi_{P}$ in $\mathbb{Q}$. It is non-positive [19, Theorem 9.1.23].

Theorem 1.1. Let $R$ be a discrete valuation ring with perfect residue field and with 2 non-zero in $R$. Let $C$ be a hyperelliptic curve of genus $g \geq 2$ with semistable reduction over $K=F r(R)$ and let $X / S$ be the minimal regular model of $C$ over $S=\operatorname{Spec} R$. Assume that all Weierstrass points of $C$ are rational over $K$. Then the integers $d_{X / S}$ and $\delta_{X / S}$ satisfy the following relation:

$$
(3 g-1) d_{X / S}=-\frac{1}{2} \sum_{P \in F(R)} \Phi_{P}^{2}+(2 g-1)(g+1) \delta_{X / S}+4\left(E, \omega_{X / S}\right) .
$$

Here $E$ is the vertical part of the divisor of the Wronskian on an R-basis of $H^{0}\left(X, \omega_{X / S}\right)$.

The divisor $E$ will be explained in more detail in Sect. 2. In Sect. 3.4 we will give an explicit formula for $E$. The divisor $\Phi_{P}$ can be effectively calculated by solving a system of linear equations with rational coefficients. The assumption on the rationality of all Weierstrass points already almost guarantees that $C$ has semistable reduction over $K$ (cf. for a precise statement the beginning of Section 4 of [14] and Sect. 3.4 below). Our theorem has the following global counterpart.

Corollary 1.2. Let $S$ be a smooth proper connected curve over an algebraically closed field $k$ such that 2 is invertible in $k$. Let $\rho: X \rightarrow S$ be a semistable hyperelliptic curve of genus $g \geq 2$ with $X$ regular. Assume that all Weierstrass points of the generic fiber of $X / S$ are rational over the function field of $S$. Then:

$$
\begin{aligned}
(3 g-1)(8 g+4) \operatorname{deg} \operatorname{det} R \rho_{*} \omega_{X / S}= & -\frac{1}{2} \sum_{P \in F(S)} \Phi_{P}^{2}+(2 g-1)(g+1) \\
& \times \sum_{\wp \in|S|} \delta_{\wp}+4\left(E, \omega_{X / S}\right),
\end{aligned}
$$

where for each closed point $\wp$ of $S, \delta_{\wp}$ denotes the number of singular points in the fiber of $X / S$ at $\wp$. The divisor $E$ is defined by taking locally over $S$ the vertical part of the divisor of the Wronskian on a basis of the locally free $O_{S}$-module $\rho_{*} \omega_{X / S}$. The divisor $\Phi_{P}$ is vertical as well and is defined as in the local case.

The proof of Theorem 1.1 will consist of a delicate sequence of specialisation and generalisation arguments. The basic point will be a comparison of the line bundles associated to $d$ and $\delta$ on the moduli stack of stable hyperelliptic curves.

A part of what we have to say about Wronskians and Weierstrass points in families carries over to the more general case of an arbitrary semistable curve over, say, a Dedekind scheme. In Sect. 4 we discuss this point in an Arakelov context. We obtain a remarkable closed formula for the stable Faltings height of a curve 
over a number field, as well as a lower bound for the self-intersection of its relative dualising sheaf. Such lower bounds are of interest in view of an effective version of the Bogomolov conjecture [32].

In this paper, all schemes are assumed locally noetherian and all morphisms of schemes are of finite type. A morphism $\rho: X \rightarrow S$ is called a prestable curve of genus $g$ if $\rho$ is proper and flat and the geometric fibers of $\rho$ are connected, reduced, nodal curves of arithmetic genus $g$. We call a prestable curve semistable (resp. stable) if every smooth rational component of a geometric fiber meets the other components of that fiber in at least 2 (resp. 3) points.

\section{Weierstrass points}

We begin by recalling from [1, Section 3] and [34, Section 2] a basic construction related to Weierstrass points in families. Let $X, S$ be regular locally noetherian schemes and let $\rho: X \rightarrow S$ be a prestable curve of genus $g \geq 1$. Let $\omega=\omega_{X / S}$ be the relative dualising sheaf of $X / S$ and let $\lambda$ be the determinant line bundle det $\rho_{*} \omega$ on $S$. Then we claim that the line bundle $\omega^{\otimes g(g+1) / 2} \otimes \rho^{*} \lambda^{-1}$ on $X$ has a canonical global section $W r$. We proceed as follows. Let $X^{s m}$ be the open subset of $X$ where $\rho$ is smooth. Let $x$ be a closed point on $X^{s m}$ and suppose it maps to a closed point $s$ of $S$. Let $t$ be a fiber coordinate on an open neighbourhood $U$ of $x$ in $X^{s m}$. Denote by $\partial^{i}$ for $i \geq 0$ the $\hat{O}_{S, s}$-linear selfmap of $\hat{O}_{X, x}=\hat{O}_{S, s}[[t]]$ given by sending $t^{n}$ to $\left(\begin{array}{c}n \\ i\end{array}\right) t^{n-i}$. If $O_{S, s}$ is of characteristic 0 then $\partial^{i}$ is just the map sending $f$ to $\frac{1}{i !} \frac{d^{i} f}{d t^{i}}$. For $\left(f_{1}, \ldots, f_{g}\right)$ in $O_{X, x}^{g}$ we let:

$$
W r\left(f_{1}, \ldots, f_{g}\right)=\operatorname{det}\left(\partial^{i-1} f_{j}\right)_{1 \leq i, j \leq g},
$$

i.e. the Wronskian of $\left(f_{1}, \ldots, f_{g}\right)$. Now let $\left(\eta_{1}, \ldots, \eta_{g}\right)$ be a basis of $\rho_{*} \omega$ around $s$ on $S$ (recall that $\rho_{*} \omega$ is locally free of rank $g$ ), and write $\eta_{i}=f_{i} \cdot d t$ with $f_{i}$ in $O_{X, x}$. If we then put $W r\left(\eta_{1}, \ldots, \eta_{g}\right)=W r\left(f_{1}, \ldots, f_{g}\right) \cdot(d t)^{\otimes g(g+1) / 2}$ it can be checked that $W r\left(\eta_{1}, \ldots, \eta_{g}\right)$ is independent of the chosen parameter $t$ and defines a section of $\omega^{\otimes g(g+1) / 2}$ locally around $x$. If $\left(\eta_{1}^{\prime}, \ldots, \eta_{g}^{\prime}\right)$ is another basis of $\rho_{*} \omega$ around $s$ connected to $\left(\eta_{1}, \ldots, \eta_{g}\right)$ via an invertible linear transformation $\left(a_{i j}\right)$ then one easily verifies that $\operatorname{Wr}\left(\eta_{1}^{\prime}, \ldots, \eta_{g}^{\prime}\right)=\operatorname{det}\left(a_{i j}\right) \operatorname{Wr}\left(\eta_{1}, \ldots, \eta_{g}\right)$. This implies that if we take $\operatorname{Wr}\left(\eta_{1}, \ldots, \eta_{g}\right) \cdot\left(\eta_{1} \wedge \ldots \wedge \eta_{g}\right)^{-1}$ we obtain a local section of $\omega^{\otimes g(g+1) / 2} \otimes \rho^{*} \lambda^{-1}$ that is independent of the choice of a basis. As we can glue these local sections over $X^{s m}$, and extend uniquely over $X$, we obtain a canonical section $W r$ of $\omega^{\otimes g(g+1) / 2} \otimes \rho^{*} \lambda^{-1}$ as required. We call $W r$ the Wronskian of $X / S$. For $X / S$ smooth the construction of the Wronskian commutes with étale base change. If $S=$ Spec $k$ where $k$ is a field and $W r$ is not identically zero we call the divisor div $W r$ of $W r$ the divisor of Weierstrass points of $X / S$. In general, if the Wronskian is not identically zero, we call the closure in $X$ of the Weierstrass points of the general fibers of $X / S$ the Weierstrass divisor of $X / S$ and denote it by $W$. We can then write $\operatorname{div} W r=W+E$ for some effective divisor $E$ on $X$ where we call $E$ 
the residual divisor of $X / S$. Both $W$ and $E$ can be viewed as Cartier divisors on $X$. The next theorem slightly generalises Lemma 3.3 of [1] and Theorem 2.10 of [34].

Proposition 2.1. (Arakelov) Let $\rho: X \rightarrow S$ be a prestable curve of genus $g \geq 1$ with $X, S$ regular locally noetherian schemes. Let $W r$ be the Wronskian of $X / S$. If $W r$ is not identically zero there exists a canonical isomorphism:

$$
\omega_{X / S}^{\otimes g(g+1) / 2} \otimes \rho^{*}\left(\operatorname{det} \rho_{*} \omega_{X / S}\right)^{-1} \stackrel{\sim}{\longrightarrow} O_{X}(W+E)
$$

of line bundles on $X$ with $W$ the Weierstrass divisor of $X / S$ and with $E$ the residual divisor of $X / S$. E has support in the union of the non-smooth fibers of $X / S$ and the fibers of positive characteristic $p$ with $p<2 g-1$. For $X / S$ smooth the formation of the isomorphism commutes with any étale surjective base change.

Proof. All statements are immediate from our discussion, except perhaps for the point that for $X / S$ smooth, the divisor $E$ may have support in the fibers of positive characteristic $p$ with $p<2 g-1$. For this we may assume that $S=\operatorname{Spec} k$ with $k$ an algebraically closed field. Let $p$ be the characteristic of $k$. We would like to prove that if $W r$ is zero, then $p$ is positive with $p<2 g-1$. Let $x$ be a closed point of $X$, and let $G(x)=\left\{a_{1}<a_{2}<\cdots\right\}$ be the set of natural numbers $a$ such that there is an $\eta$ in $H^{0}(X, \omega)$ with a zero of exact order $a-1$ at $x$. Then $G(x)$ gives rise to a filtration with simple quotients of the $g$-dimensional $k$-vector space $H^{0}(X, \omega)$, so that $G(x)$ consists of $g$ elements. Since $\omega$ has no base points we have $a_{1}=1$. On the other hand we have $a_{g} \leq 2 g-1$. Moreover there is a local parameter $t$ around $x$ and a basis $\left(f_{1} d t, \ldots, f_{g} d t\right)$ of $H^{0}(X, \omega)$ such that $f_{i}=$ (unit) $\cdot t^{a_{i}-1}$ for $i=1, \ldots, g$. This implies that there is an element $A$ in the image of $\mathbb{Z}$ in $O_{X, x}$ such that:

$$
\begin{aligned}
A \cdot W r\left(f_{1}, \ldots, f_{g}\right)= & \left|\begin{array}{cccc}
t^{a_{1}-1} & \left(a_{1}-1\right) t^{a_{1}-2} & \ldots & \left(a_{1}-1\right) \cdots\left(a_{1}-g+1\right) t^{a_{1}-g} \\
t^{a_{2}-1} & \left(a_{2}-1\right) t^{a_{2}-2} & \ldots & \left(a_{2}-1\right) \cdots \\
\vdots & \vdots & & \left(a_{2}-g+1\right) t^{a_{2}-g} \\
t^{a_{g}-1} & \left(a_{g}-1\right) t^{a_{g}-2} & \ldots & \left(a_{g}-1\right) \cdots\left(a_{g}-g+1\right) t^{a_{g}-g}
\end{array}\right| \\
& \cdot\left(\begin{array}{cccc}
\text { unit }) & & & \vdots \\
1 & \left(a_{1}-1\right) & \ldots & \left(a_{1}-1\right) \cdots\left(a_{1}-g+1\right) \\
1 & \left(a_{2}-1\right) & \ldots & \left(a_{2}-1\right) \cdots\left(a_{2}-g+1\right) \\
\vdots & \vdots & & \vdots \\
1 & \left(a_{g}-1\right) & \ldots & \left(a_{g}-1\right) \cdots\left(a_{g}-g+1\right)
\end{array} \mid \cdot(\text { unit }) \cdot t^{w}\right. \\
= & \prod_{1 \leq i<j \leq g}\left(a_{i}-a_{j}\right) \cdot(\text { unit }) \cdot t^{w}
\end{aligned}
$$

where $w=\sum_{i=1}^{g}\left(a_{i}-i\right)$. Thus if $W r\left(f_{1} d t, \ldots, f_{g} d t\right)$ is zero, so is $\prod_{1 \leq i<j \leq g}$ $\left(a_{i}-a_{j}\right)$, whence $k$ of positive characteristic $p$ with $p<2 g-1$.

The divisor $W$ is in general not finite over $S$, nor is the decomposition of $\operatorname{div} W r$ as $W+E$ in general well-behaved under base change (cf. [34, Remark 2.11]). The situation is better if $\operatorname{dim} S=1$ or if $X / S$ is a semistable hyperelliptic curve (for which see the next section). 


\section{Hyperelliptic curves}

\subsection{Preliminaries}

We begin by defining precisely what we mean by a smooth hyperelliptic curve over a base scheme $S$. A detailed account can be found in [17]. Let $k$ be an algebraically closed field, and let $X / k$ be a proper smooth curve. We call $X / k$ hyperelliptic if the genus $g$ of $X$ is $\geq 2$ and there is an involution $\sigma$ in $\operatorname{Aut}_{k}(X)$ such that $X /\langle\sigma\rangle \cong \mathbb{P}^{1}$. Such an automorphism is then unique. If $S$ is a locally noetherian scheme and $\rho: X \rightarrow S$ is a smooth proper curve we say that $X / S$ is hyperelliptic if there exists an involution $\sigma$ in $\operatorname{Aut}_{S}(X)$ such that the restriction of $\sigma$ to each geometric fiber of $\rho$ gives that fiber the structure of a hyperelliptic curve (cf. [17, Definition 5.4 and Theorem 5.5]). We note that the involution $\sigma$ is uniquely determined since the automorphism scheme $\underline{\operatorname{Aut}}_{S}(X)$ is unramified over $S$ ([9, Theorem 1.11]). We call $\sigma$ the hyperelliptic involution of $X / S$. If $X / S$ is a smooth hyperelliptic curve, we denote by $F=F_{X / S}$ the fixed point subscheme of $X$ under the action of the group $\langle\sigma\rangle$. By [17, Proposition 6.3] the scheme $F$ is the closed subscheme associated to an effective Cartier divisor on $X$ relative to $S$. The divisor $F$ is finite and flat over $S$ of degree $2 g+2$, and its formation commutes with arbitrary base change.

Lemma 3.2. Assume that $S$ is a regular locally noetherian scheme, and that $X / S$ is a smooth hyperelliptic curve of genus $g$. Then $X$ is regular, the Wronskian $W r$ of $X / S$ is not identically zero, the residual divisor $E$ of $X / S$ is empty, and the equality of Cartier divisors $W=g(g-1) / 2 \cdot F$ holds.

Proof. That $X$ is regular follows from [19, Theorem 4.3.36]. For the rest it suffices to consider the case $S=$ Spec $k$ with $k$ an algebraically closed field. Then an affine part of $X / S$ can be given by an equation $y^{2}+a y=b$ with $a, b$ in $k[x]$. The hyperelliptic involution $\sigma$ is given by $y \mapsto-y-a$. As $X /\langle\sigma\rangle$ has genus 0 , the quotient map $X \rightarrow X /\langle\sigma\rangle$ is separable. This implies that $2 y+a$ is not identically zero, and that a basis of the regular differentials is given by $x^{i} \mathrm{~d} x /(2 y+a)$ for $i=0, \ldots, g-1$. A computation yields:

$$
W r\left(\frac{\mathrm{d} x}{2 y+a}, \ldots, \frac{x^{g-1} \mathrm{~d} x}{2 y+a}\right)=(2 y+a)^{g(g-1) / 2}\left(\frac{\mathrm{d} x}{2 y+a}\right)^{\otimes g(g+1) / 2},
$$

which is not identically zero. It follows that $E$ is empty and finally, since $F$ is locally given by the vanishing of $2 y+a$, the equality $W=g(g-1) / 2 \cdot F$.

If $\rho: X \rightarrow S$ is an arbitrary proper flat curve with $S$ locally noetherian, we call $X / S$ hyperelliptic if there exists an involution $\sigma$ in $\operatorname{Aut}_{S}(X)$ and an open dense subset $U$ of $S$ such that the restriction of $\rho$ to $X \times{ }_{S} U$ is a smooth hyperelliptic curve with the restriction of $\sigma$ to $X \times{ }_{S} U$ as hyperelliptic involution. We remark that if $S$ is a connected regular noetherian scheme of dimension 1 and $X$ is the regular minimal or stable model of its generic fiber, then $X / S$ is hyperelliptic if and only if its generic fiber is hyperelliptic. Indeed, a hyperelliptic involution of the generic fiber extends by the valuative criterion of properness, and according to [17, Proposition 5.14] the smooth fibers of $\rho$ are then organised in a smooth 
hyperelliptic curve over an open dense subset of $S$. We denote by $F$ the closure in $X$ of the fixed point subscheme on the smooth fibers of $X / S$.

Let $S$ again be arbitrary. Let $\rho: X \rightarrow S$ be hyperelliptic of genus $g \geq 2$, and assume $X$ to be regular. Let $\omega_{X / S}$ be the relative dualising sheaf of $X / S$. The line bundle $\left(\operatorname{det} \rho_{*} \omega_{X / S}\right)^{\otimes 8 g+4}$ on $S$ has then a canonical non-zero global section $\Lambda$, called the hyperelliptic discriminant of $X / S$ [14, Section 2]. For $S=\operatorname{Spec} R$ with $R$ a discrete valuation ring with 2 non-zero in $R$ it can be defined as follows. Let $K=F r(R)$, and let $y^{2}=f(x)$ with $f$ in $K[x]$ monic separable of degree $2 g+2$ be an equation for $X_{K}$. Then we put:

$$
\Lambda=\left(2^{-(4 g+4)} \cdot D(f)\right)^{g} \cdot\left(\frac{\mathrm{d} x}{y} \wedge \cdots \wedge \frac{x^{g-1} \mathrm{~d} x}{y}\right)^{\otimes 8 g+4}
$$

where $D(f)$ is the discriminant of $f$ in $K$. The section $\Lambda$ is independent of the choice of $f$ and hence defines a non-zero canonical element of $H^{0}\left(X_{K}, \Omega_{X_{K}}^{1}\right)^{\otimes 8 g+4}$. It can then be viewed as a rational section of $\left(\operatorname{det} \rho_{*} \omega_{X / S}\right)^{\otimes 8 g+4}$ over $S$. The integer $d_{X / S}$ is defined to be the valuation $\operatorname{ord}_{v}(\Lambda)$ of $\Lambda$ at the closed point $v$ of $S$. The construction of $\Lambda$ generalises over arbitrary locally noetherian base schemes [21, Proposition 2.7]. For $X / S$ smooth the section $\Lambda$ is nowhere vanishing on $S$.

\subsection{Proof of Theorem 1.1}

The proof of Theorem 1.1 goes by a number of specialisation and generalisation arguments. Whenever $\rho: X \rightarrow S$ is a semistable curve, we just write $\omega$ for its relative dualising sheaf $\omega_{X / S}$ and $\lambda$ for its determinant line bundle det $R \rho_{*} \omega$. The formation of both $\omega$ and $\lambda$ commutes with arbitrary base change. When dealing with the tensor product of line bundles, we will often mix additive and multiplicative notation; we hope that this does not lead to confusion.

We start with an arbitrary generically smooth semistable curve $\rho: X \rightarrow S$ of genus $g \geq 2$ with $S$ locally noetherian. We consider the line bundle $Q=\left\langle(2 g-1)(g+1) \omega-4 \rho^{*} \lambda, \omega\right\rangle$ on $S$; here $\langle L, M\rangle$ denotes the Deligne symbol of two line bundles $L, M$ on $X$ [8, Sections 6 and 7] [24, Section 5.4]. The Deligne symbol is a biadditive functorial pairing from the category of line bundles on $X$ to the category of line bundles on $S$. For $L=\rho^{*} N$ and for $M$ flat over $S$ one has $\langle L, M\rangle=\left\langle\rho^{*} N, M\right\rangle \stackrel{\sim}{\longrightarrow} N^{\otimes d}$ canonically, where $d$ is the degree of $M$ in the fibers of $\rho$. In our case we obtain a canonical isomorphism of line bundles $Q \stackrel{\sim}{\longrightarrow}(2 g-1)(g+1)\langle\omega, \omega\rangle-(8 g-8) \lambda$ on $S$. According to [23, Théorème 2.1] we also have a canonical isomorphism of line bundles $\langle\omega, \omega\rangle \stackrel{\sim}{\longrightarrow} 12 \lambda-\delta$ on $S$, where $\delta$ is the line bundle on $S$ given by the boundary divisor on the moduli stack of stable curves of genus $g$ or, equivalently, the zero divisor of the section $\Delta$ coming from the Mumford isomorphism over the locus of $S$ where $\rho$ is smooth. A combination of these two isomorphisms yields a canonical isomorphism $Q \stackrel{\sim}{\longrightarrow}(3 g-1)(8 g+4) \lambda-(2 g-1)(g+1) \delta$ of line bundles on $S$. Note 
that all isomorphisms are compatible with any base change preserving the generic smoothness of $\rho$.

Next we impose on $\rho$ the condition that it is hyperelliptic, and on $S$ the condition that it is both reduced and irreducible, and that it has a non-empty open subscheme $U$ which is both regular and a scheme over $\mathbb{Z}[1 / 2]$. We claim that under these assumptions the line bundle $Q^{\otimes 2}$ has a canonical non-zero rational section $\xi$, having the property that its valuation $\operatorname{ord}_{v}(\xi)$ at the closed point $v$ of the spectrum $S$ of a discrete valuation ring equals $\operatorname{ord}_{v}(\xi)=-\sum_{P \in F(S)} \Phi_{P}^{2}+8(E, \omega)$, if all Weierstrass points on the generic fiber of $X / S$ are rational, and $X$ is the regular minimal model of its generic fiber. As $S$ is reduced and irreducible, it suffices to construct $\xi$ over the non-empty open subscheme $U$. By shrinking $U$ if necessary, we may assume that $\rho$ is smooth over $U$. Hence we reduce to the case that $\rho$ is smooth over $S$ and that $S$ is regular and a scheme over $\mathbb{Z}[1 / 2]$. In this case $X$ itself is regular by Lemma 3.2 and the fixed point subscheme $F$ of $X$ over $S$ is finite étale of degree $2 g+2$ over $S$ by [17, Corollary 6.8]. After a faithfully flat base change we can assume that $F$ is the disjoint union of $2 g+2$ sections of $S$. By faithfully flat descent we may assume that this is then already the case. Thus, let $P$ be a section of $F$ over $S$. By [7, Lemma 6.2] we have a unique isomorphism $\omega \stackrel{\sim}{\longrightarrow}(2 g-2) O_{X}(P) \otimes \rho^{*}\langle P, P\rangle^{\otimes-(2 g-1)}$ of line bundles on $X$ that induces, by pulling back along $P$, the canonical adjunction isomorphism $\langle P, \omega\rangle \stackrel{\sim}{\longrightarrow}\langle P, P\rangle^{\otimes-1}$ (cf. [22, Théorème 6.11]). The formation of this isomorphism commutes with arbitrary base change. Pairing the isomorphism with $\omega$ and using adjunction one obtains a canonical isomorphism $\langle\omega, \omega\rangle \stackrel{\sim}{\longrightarrow} 4 g(g-1)\langle P, \omega\rangle$ of line bundles on $S$. Taking the sum over all $P$ we obtain, using that $W=g(g-1) / 2 \cdot F$ by Lemma 3.2, a canonical isomorphism $\langle 4 W-(g+1) \omega, \omega\rangle^{\otimes 2} \stackrel{\sim}{\longrightarrow} O_{S}$ of line bundles on $S$. With Lemma 2.1 we get, as $E$ is empty in the present case by Lemma 3.2, a canonical isomorphism $Q^{\otimes 2}=\left\langle(2 g-1)(g+1) \omega-4 \rho^{*} \lambda, \omega\right\rangle^{\otimes 2} \stackrel{\sim}{\longrightarrow} O_{S}$. We get $\xi$ by taking the section of $Q^{\otimes 2}$ that corresponds to the canonical section 1 of $O_{S}$ under this isomorphism.

Now assume that $S$ is the spectrum of a discrete valuation ring, that all Weierstrass points on the generic fiber of $X / S$ are rational, and that $X$ is the regular minimal model of its generic fiber. It follows from the construction of $\xi$ above that for each $P \in F(S)$ we have a canonical trivialising section $s_{P}$ of the line bundle $\omega-(2 g-2) O_{X}(P)+\rho^{*}\langle P, P\rangle^{\otimes 2 g-1}$ restricted to the generic fiber of $X / S$. This section extends uniquely to a rational section $s_{P}$ of this same line bundle over $X$. Let's denote by $\Phi_{P}^{\prime}$ its divisor. Then $\Phi_{P}^{\prime}$ is supported on the special fiber of $X / S$ and we have $P^{*} \Phi_{P}^{\prime}$ trivial on $S$ since the pullback of our line bundle along $P$ is $\langle P, P \otimes \omega\rangle$ which is canonically trivial by adjunction. It follows easily that $\Phi_{P}^{\prime}$ is equal to $\Phi_{P}$. Thus we have a canonical isomorphism $\omega-(2 g-2) O_{X}(P)+\rho^{*}\langle P, P\rangle^{\otimes 2 g-1} \stackrel{\sim}{\longrightarrow} O_{X}\left(\Phi_{P}\right)$ of line bundles on $X$. Pairing with $\omega$ yields a canonical isomorphism $-4 g(g-1)\langle P, \omega\rangle+\langle\omega, \omega\rangle \stackrel{\sim}{\longrightarrow}\left\langle\Phi_{P}, \omega\right\rangle$ of line bundles on $S$. On the other hand, pairing with $\Phi_{P}$ yields the isomorphism $\left\langle\Phi_{P}, \omega\right\rangle \stackrel{\sim}{\longrightarrow}\left\langle\Phi_{P}, \Phi_{P}\right\rangle$. Combining we find an isomorphism $-4 g(g-1)\langle P, \omega\rangle+\langle\omega, \omega\rangle \stackrel{\sim}{\longrightarrow}\left\langle\Phi_{P}, \Phi_{P}\right\rangle$ and summing over $P \in F(S)$ and adding $8\langle E, \omega\rangle$ on both sides gives the isomorphism 
$\langle 4 W-(g+1) \omega+4 E, \omega\rangle^{\otimes 2} \stackrel{\sim}{\longrightarrow}-\sum_{P \in F(S)}\left\langle\Phi_{P}, \Phi_{P}\right\rangle+8\langle E, \omega\rangle$. Using Lemma 2.1 we find a canonical isomorphism $Q^{\otimes 2}=\langle(2 g-1)(g+1)$ $\left.\omega-4 \rho^{*} \lambda, \omega\right\rangle^{\otimes 2} \stackrel{\sim}{\longrightarrow}-\sum_{P \in F(S)}\left\langle\Phi_{P}, \Phi_{P}\right\rangle+8\langle E, \omega\rangle$. The line bundle on the right hand side has a canonical non-zero rational section $\xi^{\prime}$ whose valuation at the closed point $v$ of $S$ equals $\operatorname{ord}_{v}\left(\xi^{\prime}\right)=-\sum_{P \in F(S)} \Phi_{P}^{2}+8(E, \omega)$. It follows from the constructions that $\xi$ in $Q^{\otimes 2}$ corresponds to $\xi^{\prime}$, and thus we find the required formula for $\xi$.

The proof of Theorem 1.1 will be done if we could also prove the formula $\operatorname{ord}_{v}(\xi)=(6 g-2) \operatorname{ord}_{v}(\Lambda)-(4 g-2)(g+1) \operatorname{ord}_{v}(\Delta)$ for the valuation at $v$ of $\xi$. For this, recall the canonical isomorphism $Q \stackrel{\sim}{\longrightarrow}(3 g-1)(8 g+4) \lambda-(2 g-1)(g+1) \delta$ of line bundles on $S$ that we obtained from Mumford's isomorphism. View $\Lambda^{\otimes 3 g-1}$ as a rational section of the right hand side of this isomorphism. We will be done once we prove that under the square of the isomorphism $Q \stackrel{\sim}{\longrightarrow}(3 g-1)(8 g+4)$ $\lambda-(2 g-1)(g+1) \delta$, the rational section $\xi$ is identified with $\Lambda^{\otimes 6 g-2}$, up to a sign. In order to accomplish this, we first prove that for each stable hyperelliptic curve $\rho: X \rightarrow S$ with $S$ locally noetherian, reduced and irreducible and having the property that it contains a non-empty open subscheme $U$ that is regular and defined over $\mathbb{Z}[1 / 2]$, the sections $\xi$ and $\Lambda^{\otimes 6 g-2}$ are identified, up to a sign. For this it suffices to consider the algebraic stack $\overline{\mathcal{I}_{g}}$ classifying stable hyperelliptic curves of genus $g$ studied in e.g. [5, Section 4b] (over $\mathbb{C}$ ) and [35, Section 1] (over $\mathbb{Z}$ ). It is a suitable compactification of the algebraic stack $\mathcal{I}_{g}$ of smooth hyperelliptic curves of genus $g$. The stack $\mathcal{I}_{g}$ has smooth and geometrically irreducible fibers over Spec $\mathbb{Z}$ (cf. [15, Theorem 3]) and is in particular itself reduced and irreducible. The same holds then for $\overline{\mathcal{I}_{g}}$ and it is clear that the latter contains a non-empty open substack which is regular and defined over $\mathbb{Z}[1 / 2]$ (just take $\mathcal{I}_{g} \otimes \mathbb{Z}[1 / 2]$ ). For any stable hyperelliptic curve $\rho: X \rightarrow S$ of genus $g$ with $S$ locally noetherian, reduced and irreducible and having a non-empty regular open subscheme defined over $\mathbb{Z}[1 / 2]$, we obtain the sections $\xi$ and $\Lambda^{\otimes 6 g-2}$ over $S$ by pullback from $\overline{\mathcal{I}_{g}}$ under the period map $S \rightarrow \overline{\mathcal{I}_{g}}$.

Now note that both $\xi$ and $\Lambda^{\otimes 6 g-2}$ are supported on the boundary of $\mathcal{I}_{g}$ in $\overline{\mathcal{I}_{g}}$. Letting $\phi$ be the square of the canonical isomorphism $Q \stackrel{\sim}{\longrightarrow}(3 g-1)(8 g+4) \lambda-$ $(2 g-1)(g+1) \delta$ over $\overline{\mathcal{I}_{g}}$ we find that $\phi(\xi) \otimes \Lambda^{\otimes-(6 g-2)}$ is a rational function on $\overline{\mathcal{I}_{g}}$, regular invertible on $\mathcal{I}_{g}$. By [7, Proposition 7.3], the function $\phi(\xi) \otimes \Lambda^{\otimes-(6 g-2)}$ equals \pm 1 . Thus the sections $\xi$ and $\Lambda^{\otimes 6 g-2}$ are identified, up to a sign, as required.

The proof will now be finished by making the transition, in the case that $S$ is the spectrum of a discrete valuation ring, from a stable model $X / S$ to its associated regular minimal model $X^{\prime} / S$ with structure morphism $\rho^{\prime}$. Let $\omega^{\prime}$ be the relative dualising sheaf of $X^{\prime} / S$, and write $\lambda^{\prime}=\operatorname{det} \rho_{*} \omega^{\prime}$ and $Q^{\prime}=\left\langle(2 g-1)(g+1) \omega^{\prime}-4 \rho^{\prime *} \lambda^{\prime}, \omega^{\prime}\right\rangle$. Let $\xi^{\prime}$ be the canonical rational section of $Q^{\prime \otimes 2}$, and let $\Lambda^{\prime}$ be the canonical rational section of $\lambda^{\prime \otimes 8 g+4}$. Finally let $\pi: X^{\prime} \rightarrow X$ be the canonical map contracting the $(-2)$-curves in the special fiber. We know that for $X / S$ the sections $\xi$ of $Q^{\otimes 2}=\left\langle(2 g-1)(g+1) \omega-4 \rho^{*} \lambda, \omega\right\rangle$ and $\Lambda^{\otimes 6 g-2}$ of $(6 g-2)(8 g+4) \lambda-(4 g-2)(g+1) \delta$ correspond, up to a sign. We have a canonical isomorphism $\pi^{*} \omega \stackrel{\sim}{\longrightarrow} \omega^{\prime}$ and for any pair of line bundles $L, M$ on $X$ a canonical 
isomorphism $\left\langle\pi^{*} L, \pi^{*} M\right\rangle \stackrel{\sim}{\longrightarrow}\langle L, M\rangle$ [24, Section 5.4]. Thus we find a canonical isomorphism $Q \stackrel{\sim}{\longrightarrow} Q^{\prime}$, and one verifies that $\xi$ and $\xi^{\prime}$ are identified in this way. Also we have a canonical isomorphism $\lambda \stackrel{\sim}{\longrightarrow} \lambda^{\prime}$, yielding an identification of $\Lambda$ and $\Lambda^{\prime}$. We conclude that $\xi^{\prime}$ and $\Lambda^{\prime \otimes 6 g-2}$ are identified as well.

\subsection{Effective computations}

In this section we indicate how, for a semistable hyperelliptic curve $X$ over a discrete valuation ring $R$ in which 2 is a unit, the residual divisor $E$ can be effectively calculated. We base our discussion on Section 4 of I. Kausz's article [14]. Another approach can be found in [34, Sect. 4]. We assume that all Weierstrass points on the generic fiber are rational. This implies (cf. [14, Lemma 4.1]) that the generic fiber of $X / R$ has an equation $y^{2}=A f(x)$ with $A$ a unit in $R$ and $f(x)=\prod_{i=1}^{2 g+2}\left(x-a_{i}\right)$ for certain pairwise distinct $a_{i} \in R$. At the expense of making a small finite extension of $R$ we assume that the $v\left(a_{i}-a_{j}\right)$ are even for all $i \neq j$, and that the number of distinct images of the $a_{i}$ in $R / m$ is at least 3. Here $v$ is the normalised discrete valuation of $R$, and $m$ is the maximal ideal of $R$.

Assume that $X$ is the minimal regular model of its generic fiber. We can describe $X / R$ in a combinatorial way. We start by constructing a finite tree $T=(\mathcal{V}, \mathcal{E})$ from the above data. For non-negative integers $n$ denote by $r_{n}:\left\{a_{1}, \ldots, a_{2 g+2}\right\} \rightarrow R / m^{n}$ the natural map sending $a_{i}$ to its residue class modulo $m^{n}$. The vertices of $T$ are then the elements of the set $\mathcal{V}=\sqcup_{n \geq 0} \mathcal{V}_{n}$ where $\mathcal{V}_{n}=\left\{V \in R / m^{n}: \# r_{n}^{-1}(V) \geq 2\right\}$. The set $\mathcal{E}$ of edges of $T$ consists of the pairs $\left(V, V^{\prime}\right)$ where $V \in \mathcal{V}_{n}$ and $V^{\prime} \in \mathcal{V}_{n+1}$ for some $n \geq 0$ and $V^{\prime} \mapsto V$ under the canonical map $\mathcal{V}_{n+1} \rightarrow \mathcal{V}_{n}$. It follows that $\mathcal{V}$ has a canonical partial ordering and that there is a unique minimal element $V_{0}$ with respect to this ordering. Moreover, $T$ is canonically isomorphic to the dual graph of the special fiber of the prestable curve $Y^{\prime} / R$ of genus 0 that is obtained by taking the smooth curve $\mathbb{P}_{R}^{1}$ and then successively blowing up the closed points of the special fiber where the sections $P_{i}$ given by the $a_{i}$ meet, until the strict transform of $\sum_{i} P_{i}$ in $Y^{\prime}$ becomes regular.

We can construct $X$ from $Y^{\prime}$. For every $V \in \mathcal{V}$ put $n(V)=n$ if $V \in \mathcal{V}_{n}$, and put $\varphi(V)=\# r_{n}^{-1}(V)$. Next define $C(V)$ to be 1 if both $n$ and $\varphi(V)$ are odd, and 0 otherwise. This gives rise to an effective divisor $C=\sum_{i=1}^{2 g+2} P_{i}+\sum_{V \in \mathcal{V}} C(V) \cdot V$ on $Y^{\prime}$ which has the properties that $C$ is regular and that the class of $C$ is divisible by 2 in the Picard group of $Y^{\prime}$. By standard constructions we obtain an $R$-scheme $X^{\prime}$ and a finite flat morphism $\pi^{\prime}: X^{\prime} \rightarrow Y^{\prime}$ of degree 2 such that $X^{\prime}$ is regular and $\pi^{\prime}$ is branched exactly along $C$. In fact $X^{\prime} / R$ is prestable with generic fiber isomorphic to the generic fiber of $X$. For $V$ an irreducible component of the special fiber of $Y^{\prime}$, set $\widetilde{V}=\pi^{\prime *} V=X^{\prime} \times{ }_{Y^{\prime}} V$. If $C(V)=1$ then $\widetilde{V}=2 L$ with $L$ an exceptional smooth rational curve. If $C(V)=0$ then $\widetilde{V}$ is reduced and $\widetilde{V} \rightarrow V$ is finite of degree 2, ramified over precisely the $(V, C)$ intersection points of $C$ with $V$. Upon contracting all exceptional smooth rational curves in the special fiber of $X^{\prime}$ we find the regular semistable model $X / R$, up to $R$-isomorphism. Likewise, one can contract all components $V$ on $Y^{\prime}$ with $C(V)=1$. We denote the resulting model by $Y$, and we have a canonical map $\pi: X \rightarrow Y$ of $R$-schemes. 
Proposition 3.5. Let $X$ be a hyperelliptic semistable curve of genus $g \geq 2$ over a discrete valuation ring $R$ in which 2 is a unit. Assume that $X$ is regular. Suppose that the generic fiber of $X / R$ is given by an equation $y^{2}=A \cdot f(x)$ with $A \in R^{*}$ and $f(x)=\prod_{i=1}^{2 g+2}\left(x-a_{i}\right)$ for certain distinct $a_{i} \in R$. Assume that $v\left(a_{i}-a_{j}\right)$ is even for $i \neq j$, and that the number of distinct images of the $a_{i}$ in $R / m$ is at least 3 . Let $T=(\mathcal{V}, \mathcal{E})$ be the tree associated to the $a_{i}$ as above, and let e be the integer:

$$
e=\frac{1}{2} \sum_{V>V_{0}: \varphi(V) \text { even }} \frac{\varphi(V)}{2}\left(\frac{\varphi(V)}{2}-1\right)+\frac{1}{2} \sum_{V>V_{0}: \varphi(V) \text { odd }}\left(\frac{\varphi(V)-1}{2}\right)^{2} .
$$

Here the sums run over $V$ in the vertex set $\mathcal{V}$. Then for the residual divisor $E$ of $X / R$ one has the formula:

$$
E=\sum_{\substack{V \in \mathcal{V} \\ C(V)=0}}\left(e-\frac{g}{2} \sum_{i=1}^{n(V)} \varphi\left(V_{i}\right)+\frac{g(g+1)}{2} n(V)\right) \cdot \widetilde{V},
$$

where for each given $V$ in $\mathcal{V}$ we denote by $V_{0}, V_{1}, \ldots, V_{n}=V$ the vertices of the unique linear subgraph of $T$ that connects $V$ and $V_{0}$.

Proof. The divisor $E$ is the vertical part of the divisor of the Wronskian $W r\left(\omega_{0}, \ldots, \omega_{g-1}\right)$ on an $R$-basis $\left(\omega_{0}, \ldots, \omega_{g-1}\right)$ of $H^{0}(X, \omega)$. According to [14, Proposition 5.5] there are $e_{i} \in \mathbb{Z}$ with $\sum_{i=0}^{g-1} e_{i}=e$ and $b_{j} \in\left\{a_{1}, \ldots, a_{2 g+2}\right\}$ such that for:

$$
\omega_{i}=t^{e_{i}}\left(\prod_{j=1}^{i}\left(x-b_{j}\right)\right) \frac{\mathrm{d} x}{y}, \quad i=0, \ldots, g-1
$$

the tuple $\left(\omega_{0}, \ldots, \omega_{g-1}\right)$ is an $R$-basis of $H^{0}(X, \omega)$. Here $t$ is a generator of the maximal ideal $m$ of $R$. Putting $h_{i}(x)=\prod_{j=1}^{i}\left(x-b_{j}\right)$ for $i=0, \ldots, g-1$ a computation shows that:

$$
\begin{aligned}
W r\left(\omega_{0}, \ldots, \omega_{g-1}\right) & =y^{-g} t^{e} W r\left(h_{0}, \ldots, h_{g-1}\right)(\mathrm{d} x)^{\otimes g(g+1) / 2} \\
& =y^{g(g-1) / 2} t^{e} W r\left(h_{0}, \ldots, h_{g-1}\right)\left(\frac{\mathrm{d} x}{y}\right)^{\otimes g(g+1) / 2} \\
& =y^{g(g-1) / 2} t^{e}\left(\frac{\mathrm{d} x}{y}\right)^{\otimes g(g+1) / 2}
\end{aligned}
$$

From this we compute $\operatorname{div} \operatorname{Wr}\left(\omega_{0}, \ldots, \omega_{g-1}\right)$. Let $\operatorname{div}_{\text {vert }} y$ be the vertical part of the divisor of $y$ on $X$, and let $P_{\infty}$ be the section of $Y$ corresponding to the point at infinity of the generic fiber of $Y$. According to Lemma 5.2 of [14] we have:

$$
\operatorname{div}\left(\frac{\mathrm{d} x}{y}\right)=(g-1) \pi^{*} P_{\infty}-\operatorname{div}_{\mathrm{vert}} y+\sum_{\substack{V \in \mathcal{V} \\ C(V)=0}} n(V) \cdot \widetilde{V}
$$


where $\pi: X \rightarrow Y$ is the canonical map. It follows that:

$$
\begin{aligned}
\operatorname{div} W r\left(\omega_{0}, \ldots, \omega_{g-1}\right)= & e F+\frac{g(g-1)}{2} \operatorname{div} y+\frac{g(g-1)(g+1)}{2} \pi^{*} P_{\infty} \\
& -\frac{g(g+1)}{2} \operatorname{div}_{\text {vert }} y+\frac{g(g+1)}{2} \sum_{\substack{V \in \mathcal{V} \\
C(V)=0}} n(V) \cdot \widetilde{V}
\end{aligned}
$$

with $F$ the special fiber of $X$. Noting that:

$$
\frac{g(g-1)}{2} \operatorname{div} y=\frac{g(g-1)}{2} \operatorname{div}_{\text {vert }} y+W-\frac{g(g-1)(g+1)}{2} \pi^{*} P_{\infty}
$$

we derive:

$$
\operatorname{div} W r\left(\omega_{0}, \ldots, \omega_{g-1}\right)=e F+W-g \operatorname{div}_{\mathrm{vert}} y+\frac{g(g+1)}{2} \sum_{\substack{V \in \mathcal{V} \\ C(V)=0}} n(V) \cdot \widetilde{V}
$$

Clearly for each $V$ in $\mathcal{V}$ with $C(V)=0$ we have $v_{\widetilde{V}}(y)=\frac{1}{2} \sum_{i=1}^{2 g+2} v_{\widetilde{V}}\left(x-a_{i}\right)$. The valuation $v_{\widetilde{V}}\left(x-a_{i}\right)$ can be seen to be equal to $\min \left(n(V), v\left(a-a_{i}\right)\right)$ where $a$ is a representative of $V$ (cf. [14, Proof of Lemma 5.1]). A counting argument shows that $\sum_{i=1}^{2 g+2} \min \left(n(V), v\left(a-a_{i}\right)\right)=\sum_{i=1}^{n(V)} \varphi\left(V_{i}\right)$ if $V_{0}, V_{1}, \ldots, V_{n}=V$ are the vertices of the unique linear subgraph of $T$ that connects $V$ and $V_{0}$. Thus we obtain $v_{\widetilde{V}}(y)=\sum_{i=1}^{n(V)} \varphi\left(V_{i}\right)$ for each $V$ with $C(V)=0$ and we arrive at:

$\operatorname{div} W r\left(\omega_{0}, \ldots, \omega_{g-1}\right)=W+\sum_{\substack{V \in \mathcal{V} \\ C(V)=0}}\left(e-\frac{g}{2} \sum_{i=1}^{n(V)} \varphi\left(V_{i}\right)+\frac{g(g+1)}{2} n(V)\right) \cdot \widetilde{V}$

The formula follows.

Apparently, the number $e$ can be interpreted as the multiplicity in $E$ of the irreducible component of the special fiber of $X / R$ that maps to $V_{0}$ in $Y / R$. We note however that the component $V_{0}$ may depend on the particular equation chosen for the generic fiber of $X / R$.

\subsection{Example}

In this section we verify Theorem 1.1 for a concrete case with $g=2$. Let $k$ be an algebraically closed field with $2 \in k^{*}$, and let $X_{k}^{\prime}$ be a stable curve of genus 2 over $k$ consisting of an elliptic curve $A$ with a one-noded rational curve $B$ attached to it. Let $X_{k} \rightarrow X_{k}^{\prime}$ be the modification of $X_{k}^{\prime}$ obtained by partially normalising $X_{k}^{\prime}$ at the node $v$ of $B$, and attaching a projective line $D$ at the two points in the preimage of $v$. The semi-stable curve obtained in this way has type $I_{2}-I_{0}-1$ in the classification of Namikawa-Ueno [26]. Its intersection matrix is: 


\begin{tabular}{|r|r|r|r|}
\hline & $A$ & $B$ & $D$ \\
\hline$A$ & -1 & 1 & 0 \\
$B$ & 1 & -3 & 2 \\
$D$ & 0 & 2 & -2 \\
\hline
\end{tabular}

and for the arithmetic genera $p_{a}(C)$ resp. the intersections $(C, \omega)$ with $\omega$ we have:

\begin{tabular}{|r|r|r|}
\hline$C$ & $p_{a}(C)$ & $(C, \omega)$ \\
\hline$A$ & 1 & 1 \\
$B$ & 0 & 1 \\
$D$ & 0 & 0 \\
\hline
\end{tabular}

Assume that $X_{k}$ is the special fiber of a semistable hyperelliptic curve $X / S$ with $S$ the spectrum of a discrete valuation ring $R$ with residue field $k$ and with $X$ regular. Assume that the Weierstrass points $P_{1}, \ldots, P_{6}$ of the generic fiber are rational, and that their closures in $X$ are distributed over the special fiber as follows: $P_{1}, P_{2}, P_{3}$ intersect $A, P_{4}$ intersects $B$, and $P_{5}, P_{6}$ intersect $D$. The surface $X$ is then the minimal regular model of a genus 2 curve given by an equation $y^{2}=\left(x-a_{1}\right) \cdots\left(x-a_{6}\right)$ with $a_{1}, \ldots, a_{6} \in R$ giving rise to a linear tree $V_{0}-V_{1}-V_{2}-V_{3}$ with $V_{0}$ represented by $a_{1}, \ldots, a_{6}, V_{1}, V_{2}$ represented by $a_{4}, a_{5}, a_{6}$ and $V_{3}$ represented by $a_{5}, a_{6}$. Thus $\varphi\left(V_{1}\right)=\varphi\left(V_{2}\right)=3$ and $\varphi\left(V_{3}\right)=2$. The correspondence with the $P_{i}$ is via $P_{i} \leftrightarrow a_{i}$, the component $A$ corresponds to $V_{0}$, the component $B$ corresponds to $V_{2}$ and the component $D$ corresponds to $V_{3}$. The vertex $V_{1}$ has $C(V)$ equal to 1 . We compute $e=1$ and Proposition 3.5 gives $E=A+B+2 D$ which is indeed effective.

Next, solving the equation $(2 P-\omega+\Phi, C)=0$ for $C=A, B, D$ and demanding that $(\Phi, P)=0$ one finds $\Phi_{P}$ and hence $\Phi_{P}^{2}$ for all $P \in F(S)$. The results are in the following table:

\begin{tabular}{|r|r|r|}
\hline$P$ & $\Phi_{P}$ & $\Phi_{P}^{2}$ \\
\hline$P_{1}, P_{2}, P_{3}$ & $-B-D$ & -1 \\
$P_{4}$ & $-A$ & -1 \\
$P_{5}, P_{6}$ & $-2 A-B$ & -3 \\
\hline
\end{tabular}

The formula in Theorem 1.1 reads in our case:

$$
5 d_{X / S}=-\frac{1}{2} \sum_{P \in F(R)} \Phi_{P}^{2}+9 \delta_{X / S}+4\left(E, \omega_{X / S}\right) .
$$

From $E=A+B+2 D$ we obtain $\left(E, \omega_{X / S}\right)=2$. From the tables we further read off that $-\sum_{P \in F(S)} \Phi_{P}^{2}=10$. Our description of the special fiber gives $\delta_{X / S}=3$. Finally, one has $d_{X / S}=8$ by either [14, Theorem 3.1] or [33, Table 5] (but note that in the latter reference, the hyperelliptic discriminant is a section of $\lambda^{\otimes 10}$, not 
of $\lambda^{\otimes 20}$ as in our set-up, so that to get the values for our $d$, the values for $d$ given in the table in [33] should be multiplied by 2). The formula checks.

\section{Weierstrass points on arithmetic surfaces}

In this section we consider Arakelov versions of several of the isomorphisms considered earlier, associated with Weierstrass points. Let $S$ be a connected Dedekind scheme with generic residue characteristic equal to 0 and let $\rho: X \rightarrow S$ be a semistable curve of genus $g \geq 1$. Assume that $X$ is the regular minimal model of its generic fiber. Under these assumptions, the Wronskian $W r$ of $X / S$ is not identically zero and we have a Weierstrass divisor $W$ flat over $S$ and a residual divisor $E$ supported in the non-smooth fibers of $X / S$ and in the fibers of positive characteristic $p$ with $p<2 g-1$. If $\omega$ is the relative dualising sheaf of $\rho$ and $\lambda=\operatorname{det} \rho_{*} \omega$ as before, we have a canonical isomorphism $O_{X}(W+E) \stackrel{\sim}{\longrightarrow} g(g+1) / 2 \cdot \omega-\rho^{*} \lambda$ of line bundles on $X$ (cf. Proposition 2.1). Multiplying both sides by 4 , then subtracting $(g+1) \omega$ from both sides and then pairing with $\omega$ we obtain from this a canonical isomorphism $\langle 4 W-(g+1) \omega+4 E, \omega\rangle \stackrel{\sim}{\longrightarrow}\left\langle(2 g-1)(g+1) \omega-4 \rho^{*} \lambda, \omega\right\rangle$. The latter line bundle was called $Q$ in the proof of Theorem 1.1. In that proof, we applied the Mumford isomorphism $\langle\omega, \omega\rangle \stackrel{\sim}{\longrightarrow} 12 \lambda-\delta$ to obtain a canonical isomorphism $Q \stackrel{\sim}{\longrightarrow}(3 g-1)(8 g+4) \lambda-(2 g-1)(g+1) \delta$. Combining both isomorphisms we end up with a canonical isomorphism:

$$
v:(3 g-1)(8 g+4) \lambda \stackrel{\sim}{\longrightarrow}\langle 4 W-(g+1) \omega+4 E, \omega\rangle+(2 g-1)(g+1) \delta
$$

of line bundles on $S$. Now remark that in the Faltings-Deligne version of Arakelov theory of arithmetic surfaces the line bundles under consideration on $X$ come equipped with certain canonical hermitian metrics (cf. [11, Sect. 2]), and likewise for the line bundles under consideration on $S$ (cf. [11, Sects. 3 and 4] and [8, Sect. 6]). In particular, if $S=\operatorname{Spec} \mathbb{C}$ the isomorphism $v$ has a certain norm. Our first result is that this norm is closely related to an invariant $T$ introduced in [6]. If $\mathcal{X}$ is a compact connected Riemann surface of genus $g \geq 1$, then $T(\mathcal{X})$ is given by:

$$
\begin{aligned}
T(\mathcal{X})= & \left(\frac{\|\vartheta\|\left(P_{1}+\cdots+P_{g}-P_{g+1}\right)}{\prod_{k=1}^{g}\|\vartheta\|\left(g P_{k}-P_{g+1}\right)^{1 / g}}\right)^{2 g-2} \cdot \\
& \cdot\left(\frac{\prod_{k \neq l}\|\vartheta\|\left(g P_{k}-P_{l}\right)^{1 / g}}{\|J\|\left(P_{1}, \ldots, P_{g}\right)^{2}}\right) \cdot \prod_{R \in W} \prod_{k=1}^{g}\|\vartheta\|\left(g P_{k}-R\right)^{(g-1) / g^{4}},
\end{aligned}
$$

where $P_{1}, \ldots, P_{g+1}$ are generic points on $\mathcal{X}, W$ is the divisor div $W r$ of Weierstrass points on $X,\|\vartheta\|$ is the normalised theta function on $\operatorname{Pic}^{g-1}(\mathcal{X})$ of [11, p. 401], and $\|J\|$ is a normalised jacobian determinant involving theta functions on $\operatorname{Sym}^{g}(\mathcal{X})$ introduced in [12].

Proposition 4.1. The norm of $v$ for $X$ a smooth proper curve of genus $g \geq 1$ over $S=\mathrm{Spec} \mathbb{C}$ is equal to:

$$
(2 \pi)^{-4 g(2 g-1)(g+1)} \cdot T(X(\mathbb{C}))^{8 g^{2}} .
$$


Proof. We reconstruct the isomorphism $v$ again from the isomorphism $O_{X}(W)$ $\stackrel{\sim}{\longrightarrow} g(g+1) / 2 \cdot \omega-\rho^{*} \lambda$ and the Mumford isomorphism $\langle\omega, \omega\rangle \stackrel{\sim}{\longrightarrow} 12 \lambda-\delta$, keeping track of the norms at each step (note that the residual divisor $E$ now vanishes). According to [23, Théorème 2.2] the norm of the Mumford isomorphism is equal to $(2 \pi)^{4 g} \mathrm{e}^{-\delta(X(\mathbb{C}))}$ where $\delta(X(\mathbb{C}))$ is the Faltings delta-invariant of $X(\mathbb{C})$ $\left[11\right.$, p. 402]. The inverse norm of the isomorphism $O_{X}(W) \stackrel{\sim}{\longrightarrow} g(g+1) / 2 \cdot \omega-\rho^{*} \lambda$ was called $R(X(\mathbb{C}))$ in [6] (cf. Definition 5.3 of that paper), and it follows from [6, Theorem 4.4] and its proof that $R, T$ and $\delta$ are related via:

$$
R(X(\mathbb{C}))^{g-1}=T(X(\mathbb{C}))^{g^{2}} \cdot \mathrm{e}^{-(2 g-1)(g+1) \delta(X(\mathbb{C})) / 8}
$$

(the reader is warned that the formula in Theorem 4.4 of loc. cit. contains a misprint: the $g^{3}$ occurring in the exponent should be $g^{2}$ ). From the isomorphism $O_{X}(W) \stackrel{\sim}{\longrightarrow} g(g+1) / 2 \cdot \omega-\rho^{*} \lambda$ we get by multiplying both sides by 4 , by subtracting $(g+1) \omega$ from both sides and by pairing with $\omega$ the isomorphism $\langle 4 W-(g+1) \omega, \omega\rangle \stackrel{\sim}{\longrightarrow}\left\langle(2 g-1)(g+1) \omega-4 \rho^{*} \lambda, \omega\right\rangle$ which thus has norm $R(X(\mathbb{C}))^{-(8 g-8)}$. Composing through with the isomorphism:

$$
\left\langle(2 g-1)(g+1) \omega-4 \rho^{*} \lambda, \omega\right\rangle \stackrel{\sim}{\longrightarrow}(3 g-1)(8 g+4) \lambda-(2 g-1)(g+1) \delta
$$

which has norm $\left((2 \pi)^{4 g} \mathrm{e}^{-\delta(X(\mathbb{C}))}\right)^{(2 g-1)(g+1)}$ we find the isomorphism:

$$
\langle 4 W-(g+1) \omega, \omega\rangle \stackrel{\sim}{\longrightarrow}(3 g-1)(8 g+4) \lambda-(2 g-1)(g+1) \delta
$$

having norm:

$R(X(\mathbb{C}))^{-(8 g-8)} \cdot\left((2 \pi)^{4 g} \mathrm{e}^{-\delta(X(\mathbb{C}))}\right)^{(2 g-1)(g+1)}=(2 \pi)^{4 g(2 g-1)(g+1)} \cdot T(X(\mathbb{C}))^{-8 g^{2}}$.

The norm of $v$ is the inverse of this.

Example 4.2. Let $X$ be a hyperelliptic curve of genus $g \geq 2$ over $S=\operatorname{Spec} \mathbb{C}$. It follows from the proof of Theorem 1.1 that the square of the line bundle $Q=\langle 4 W-(g+1) \omega, \omega\rangle$ on $S$ is canonically isomorphic to the trivial line bundle $O_{S}$. By going through a sequence of arguments analogous to those in the proof of Proposition 2 of [3] it can be verified that the isomorphism $Q^{\otimes 2} \stackrel{\sim}{\longrightarrow} O_{S}$ is in fact an isometry. This implies that the canonical section $\xi$ of $Q^{\otimes 2}$ has unit norm. According to [16, Section 3] and [7, p. 11] the norm of $\Lambda$ satisfies $\|\Lambda\|^{n}=(2 \pi)^{4 g^{2} r}\left\|\Delta_{g}\right\|(X(\mathbb{C}))^{g}$ where $n=\left(\begin{array}{c}2 g \\ g+1\end{array}\right)$ and $r=\left(\begin{array}{c}2 g+1 \\ g+1\end{array}\right)$ and where $\left\|\Delta_{g}\right\|(X(\mathbb{C}))$ is the Petersson norm of the modular discriminant of $X$. With Proposition 4.1 we obtain the formula $T(X(\mathbb{C}))=(2 \pi)^{-2 g} \cdot\left\|\Delta_{g}\right\|(X(\mathbb{C}))^{-\frac{3 g-1}{8 n g}}$ for the $T$-invariant of $X(\mathbb{C})$.

Proposition 4.1 can be applied to give an explicit formula for the stable Faltings height $h\left(X_{K}\right)$ of a smooth proper geometrically connected curve $X_{K}$ of genus $g \geq 2$ over a number field $K$ that has semistable reduction over $K$. Let $X$ be the regular minimal model of $X_{K}$ over $S=\operatorname{Spec} O_{K}$ and let $\omega=\omega_{X / S}$ be the relative dualising sheaf of $X / S$. For $P$ a section of $X / S$ denote as before by $\Phi_{P}$ the vertical 
$\mathbb{Q}$-divisor on $X$ such that $\left((2 g-2) P-\omega+\Phi_{P}, \Gamma\right)=0$ for all irreducible components $\Gamma$ of the geometric fibers of $X / S$ and such that $P^{*} \Phi_{P}$ is trivial. Denote by $h(P)$ the Néron-Tate height of the divisor class of $(2 g-2) P-\omega$ in the jacobian of $X_{K}$. The stable Faltings height of $X_{K}$ is defined via $[K: \mathbb{Q}] h_{F}\left(X_{K}\right)=\widehat{\operatorname{deg}} \lambda$, the latter being the Arakelov degree of $\lambda=\operatorname{det} R \rho_{*} \omega$ equipped with its canonical hermitian structure at the complex embeddings of $K$ (cf. [10, Sect. 3]).

Theorem 4.3. Assume that the Weierstrass points on the generic fiber of $X / S$ are rational over $K$. For $\wp$ a closed point of $S$ let $\delta_{\wp}$ be the number of singular points in the geometric fiber at $\wp$. The formula:

$$
\begin{aligned}
& (3 g-1)(8 g+4)[K: \mathbb{Q}] h_{F}\left(X_{K}\right) \\
& =\frac{2[K: \mathbb{Q}]}{g(g-1)} \sum_{P \in W(S)} h(P)-\frac{1}{g(g-1)} \sum_{P \in W(S)} \Phi_{P}^{2} \\
& \quad+(2 g-1)(g+1) \sum_{\wp \in|S|} \delta_{\wp} \log N \wp+4\left(E, \omega_{X / S}\right) \\
& \quad-4 g(2 g-1)(g+1)[K: \mathbb{Q}] \log (2 \pi)+8 g^{2} \sum_{\sigma: K \rightarrow \mathbb{C}} \log T\left(X_{\sigma}\right)
\end{aligned}
$$

holds. Here for each complex embedding $\sigma$ of $K$ we denote by $X_{\sigma}$ the Riemann surface corresponding to the complex curve $X \times_{\sigma} \mathbb{C}$. The intersection numbers $\Phi_{P}^{2}$ and $\left(E, \omega_{X / S}\right)$ should be taken in the Arakelov sense, that is, their local contributions at each closed point $\wp$ of $S$ should be counted with weight $\log N \wp$. In the summations over $P \in W(S)$ the Weierstrass points should be counted with their multiplicity in $W$.

Proof. We start with the canonical isomorphism:

$$
v:(3 g-1)(8 g+4) \lambda \stackrel{\sim}{\longrightarrow}\langle 4 W-(g+1) \omega+4 E, \omega\rangle+(2 g-1)(g+1) \delta
$$

of line bundles on $S$ described at the beginning of this section. The norm of this isomorphism is provided by Proposition 4.1. Taking Arakelov degrees on left and right hand side we then find:

$$
\begin{aligned}
(3 g-1)(8 g+4) \widehat{\operatorname{deg}} \lambda= & 4(W, \omega)-(g+1)(\omega, \omega) \\
& +(2 g-1)(g+1) \sum_{\wp \in|S|} \delta_{\wp} \log N \wp+4(E, \omega) \\
& -4 g(2 g-1)(g+1)[K: \mathbb{Q}] \log (2 \pi) \\
& +8 g^{2} \sum_{\sigma: K \rightarrow \mathbb{C}} \log T\left(X_{\sigma}\right)
\end{aligned}
$$

where the intersection numbers are Arakelov intersection numbers [11, Sect. 2]. According to [31, Section 1.1] we can write, for any section $P$ of $X / S$ :

$$
-2[K: \mathbb{Q}] h(P)=-4 g(g-1)(P, \omega)+(\omega, \omega)-\Phi_{P}^{2} .
$$


Thus, taking the sum over $P$ running through $W(S)$ and dividing by $g(g-1)$ we find the equality:

$$
4(W, \omega)-(g+1)(\omega, \omega)=\frac{2[K: \mathbb{Q}]}{g(g-1)} \sum_{P \in W(S)} h(P)-\frac{1}{g(g-1)} \sum_{P \in W(S)} \Phi_{P}^{2} .
$$

The required formula follows.

Upon decomposing the terms $h(P), \Phi_{P}^{2}$ and $(E, \omega)$ as sums of local contributions one may use the formula in Theorem 4.3 to arrive at a provisory definition of a 'valuation of the discriminant' $d_{\wp}$ for $X / S$ at each closed point $\wp$ of $S$. It might be worthwhile to study this 'valuation of the discriminant' further especially in the light of the fact that, as $\lambda$ is ample on the moduli stack $\mathcal{M}_{g}(\mathbb{C})$ of complex curves of genus $g \geq 3$, no positive tensor power $\lambda^{\otimes N}$ of $\lambda$ has a nowhere vanishing global section on $\mathcal{M}_{g}(\mathbb{C})$. Specialising $X / S$ to a hyperelliptic curve of genus $g$, one obtains the valuation of the hyperelliptic discriminant (up to the factor $3 g-1$ ) as follows from Theorem 1.1. An interesting discussion of the line bundle $\lambda^{\otimes 8 g+4}$ on $\mathcal{M}_{g}(\mathbb{C})$ can be found in [13].

Most of the terms in our formula for $h_{F}\left(X_{K}\right)$ can be easily bounded from below. Indeed, if $P$ is any section of $X / S$ then $h(P) \geq 0$ and $-\Phi_{P}^{2} \geq 0$. Also $(E, \omega) \geq 0$ since $E$ is effective and vertical and the fibers of $X / S$ do not contain any exceptional curves. By passing, for an arbitary curve $X_{K}$ over a number field $K$, to a finite extension $L$ of $K$ such that $X_{K}$ acquires semistable reduction over $L$ and has all its Weierstrass points rational over $L$, we obtain the following corollary.

Corollary 4.4. Let $X_{K}$ be a smooth proper geometrically connected curve of genus $g \geq 2$ over a number field $K$. Then its Faltings stable height $h_{F}\left(X_{K}\right)$ satisfies the inequality:

$$
\begin{aligned}
(3 g-1)(8 g+4) h_{F}\left(X_{K}\right) \geq & -4 g(2 g-1)(g+1) \log (2 \pi) \\
& +(2 g-1)(g+1) \frac{1}{[K: \mathbb{Q}]} \sum_{\wp \in|S|} \delta_{\wp} \log N \wp \\
& +8 g^{2} \frac{1}{[K: \mathbb{Q}]} \sum_{\sigma: K \rightarrow \mathbb{C}} \log T\left(X_{\sigma}\right) .
\end{aligned}
$$

Here again for each complex embedding $\sigma$ of $K$ we denote by $X_{\sigma}$ the Riemann surface corresponding to the complex curve $X_{K} \times_{\sigma} \mathbb{C}$.

It is perhaps interesting to point out a similarity with an inequality due to Bost [2, Theorem IV], saying that the lower bound:

$$
(8 g+4) h_{F}\left(X_{K}\right) \geq g \cdot \frac{1}{[K: \mathbb{Q}]}\left(\sum_{\wp \in|S|} \delta_{\wp} \log N \wp+\sum_{\sigma: K \rightarrow \mathbb{C}} \psi\left(X_{\sigma}\right)\right)
$$

holds, with a function $\psi: M_{g}(\mathbb{C}) \rightarrow \mathbb{R}$ which is continuous and which satisfies the following logarithmic bound around the boundary. If $X_{0}$ is a stable complex 
curve, if $\rho: X \rightarrow \Omega$ is its universal deformation with $\Omega$ an open neighbourhood of 0 in $\mathbb{C}^{3 g-3}$, and if $\delta$ is a local equation for the reduced normal crossings divisor $\Delta$ in $\Omega$ given by the singular fibers of $\rho$, then $\psi\left(X_{t}\right) \geq-\log |\delta(t)|+o(\log |\delta(t)|)$ as $t \in \Omega \backslash \Delta$ goes to 0 . The 'slope' $g /(8 g+4)$ featuring in this result is much better than the 'slope' $(2 g-1)(g+1) /(3 g-1)(8 g+4)$ of our lower bound. On the other hand the analytic contributions in our lower bound have the advantage of being more readily calculable in concrete cases, using only evaluations of theta functions and their derivatives. An asymptotic analysis of the function $\log T$ near the boundary of $M_{g}(\mathbb{C})$ was carried out in the author's thesis, showing that, similar to Bost's result, $T$ can be seen as the inverse of a distance to the boundary.

An application of the Noether formula for arithmetic surfaces [23, Théorème 2.5] yields a lower bound for the self-intersection of the relative dualising sheaf.

Corollary 4.5. Let $X_{K}$ be a smooth proper geometrically connected curve of genus $g \geq 2$ over a number field $K$. Let $e\left(X_{K}\right)$ be the normalised self-intersection of the relative dualising sheaf of $X_{K}$. Then the inequality:

$$
\begin{aligned}
(3 g-1)(8 g+4) e\left(X_{K}\right) \geq & -48 g(2 g-1)(g+1) \log (2 \pi)+\frac{8 g-8}{[K: \mathbb{Q}]} \sum_{s \in|S|} \delta_{\wp} \log N \wp \\
& +\frac{1}{[K: \mathbb{Q}]} \sum_{\sigma: K \rightarrow \mathbb{C}}\left(96 g^{2} \log T\left(X_{\sigma}\right)\right. \\
& \left.-(3 g-1)(8 g+4) \delta\left(X_{\sigma}\right)\right)
\end{aligned}
$$

holds.

Trying to obtain explicit lower bounds for $e\left(X_{K}\right)$ is interesting in the light of an effective version of the Bogomolov conjecture [32]. Lower bounds of a similar type appear in [4, Section 3.3.2], which uses more general Weierstrass points. In a recent preprint [36] Zhang has obtained explicit lower bounds for $e\left(X_{K}\right)$ assuming that a certain conjecture of Grothendieck-Gillet-Soulé on the non-negativity of the height of Gross-Schoen cycles is true.

Acknowledgments. The author thanks the Mittag-Leffler Institute in Djursholm for its hospitality during a visit. He thanks Sylvain Maugeais for several discussions related to the theme of this paper, Lidia Stoppino for pointing out the reference [2], and the anonymous referee for a number of helpful remarks. The author is supported by VENI-grant 639.031 .619 of the Netherlands Organisation for Scientific Research (NWO).

Open Access This article is distributed under the terms of the Creative Commons Attribution Noncommercial License which permits any noncommercial use, distribution, and reproduction in any medium, provided the original author(s) and source are credited.

\section{References}

[1] Arakelov, S.Y.: Families of algebraic curves with fixed degeneracies. Math. USSR Izvestija 5, 1277-1302 (1971) 
[2] Bost, J.-B.: Semi-stability and heights of cycles. Invent. Math. 118, 223-253 (1994)

[3] Bost, J.-B., Mestre, J.-F., Moret-Bailly, L.: Sur le calcul explicite des "classes de Chern" des surfaces arithmétiques de genre 2. In: Séminaire sur les pinceaux de courbes elliptiques, vol. 183, pp. 69-105. Astérisque (1990)

[4] Burnol, J.-F.: Weierstrass points on arithmetic surfaces. Invent. Math. 107, 421-432 (1992)

[5] Cornalba, M., Harris, J.: Divisor classes associated to families of stable varieties, with applications to the moduli space of curves. Ann. Sci. Ec. Num. Sup. 21, 455-475 (1988)

[6] de Jong, R.: Arakelov invariants of Riemann surfaces. Doc. Math. 10, 311-329 (2005)

[7] de Jong, R.: Explicit Mumford isomorphism for hyperelliptic curves. J. Pure Appl. Algebra 208, 1-14 (2007)

[8] Deligne, P.: Le déterminant de la cohomologie. In: Contemporary Mathematics, vol. 67, pp. 93-177. American Mathematical Society (1987)

[9] Deligne, P., Mumford, D.: The irreducibility of the space of curves of given genus. Publ. Math. de l'I.H.E.S. 36, 75-110 (1969)

[10] Faltings, G.: Endlichkeitssätze für abelsche Varietäten über Zahlkörpern. Invent. Math. 73, 349-366 (1983)

[11] Faltings, G.: Calculus on arithmetic surfaces. Ann. Math. 119, 387-424 (1984)

[12] Guàrdia, J.: Analytic invariants in Arakelov theory for curves. C.R. Acad. Sci. Paris Ser. I 329, 41-46 (1999)

[13] Hain, R., Reed, D.: On the Arakelov geometry of moduli spaces of curves. J. Differ. Geom. 67, 195-228 (2004)

[14] Kausz, I.: A discriminant and an upper bound for $\omega^{2}$ for hyperelliptic arithmetic surfaces. Compos. Math. 115, 37-69 (1999)

[15] Laudal, O.A., Lønsted, K.: Deformations of curves I. Moduli for hyperelliptic curves. Lect. Notes in Math., vol. 687, pp. 150-167. Springer (1978)

[16] Lockhart, P.: On the discriminant of a hyperelliptic curve. Trans. Am. Math. Soc. 342, 729-752 (1994)

[17] Lønsted, K., Kleiman, S.L.: Basics on families of hyperelliptic curves. Compos. Math. 38, 83-111 (1979)

[18] Liu, Q.: Conducteur et discriminant minimal de courbes de genre 2. Compos. Math. 94, 51-79 (1994)

[19] Liu, Q.: Algebraic Geometry and Arithmetic Curves. Oxford Graduate Texts in Mathematics, vol. 6. Oxford Science Publications, Oxford (2002)

[20] Matsusaka, S.: Some numerical invariants of hyperelliptic fibrations. J. Math. Kyoto Univ. 30, 33-57 (1990)

[21] Maugeais, S.: Relèvement des revêtements $p$-cycliques des courbes rationnelles semistables. Math. Ann. 327, 365-393 (2003)

[22] Moret-Bailly, L.: Métriques permises. In: Séminaire sur les pinceaux arithmétiques: la conjecture de Mordell, vol. 127, pp. 29-87. Astérisque (1985)

[23] Moret-Bailly, L.: La formule de Noether pour les surfaces arithmétiques. Inv. Math. 98, 491-498 (1989)

[24] Moret-Bailly, L.: Hauteurs et classes de Chern sur les surfaces arithmétiques. In: Séminaire sur les pinceaux de courbes elliptiques, vol. 183, pp. 37-58. Astérisque (1990)

[25] Mumford, D.: Stability of projective varieties. L'Ens. Math. 23, 33-100 (1977)

[26] Namikawa, Y., Ueno, K.: The complete classification of fibres in pencils of curves of genus two. Manuscripta Math. 9, 143-186 (1973)

[27] Ogg, A.P.: On pencils of curves of genus two. Topology 5, 353-367 (1966)

[28] Ogg, A.P.: Elliptic curves and wild ramification. Am. J. Math. 89, 1-21 (1967)

[29] Saito, T.: Conductor, discriminant, and the Noether formula of arithmetic surfaces. Duke Math. J. 57, 151-173 (1988) 
[30] Saito, T.: The discriminants of curves of genus 2. Compos. Math. 69, 229-240 (1989)

[31] Szpiro, L.: Un peu d'effectivité. In: Séminaire sur les pinceaux arithmétiques: la conjecture de Mordell, vol. 127, pp. 275-287. Astérisque (1985)

[32] Szpiro, L.: Sur les propriétés numériques du dualisant relatif d'une surface arithmétique. In: The Grothendieck Festschrift, Volume III. Progress in Mathematics, vol. 88. Birkhäuser Verlag, Basel (1990)

[33] Ueno, K.: Discriminants of curves of genus two and arithmetic surfaces. Algebraic Geometry and Commutative Algebra in Honor of Masayoshi Nagata, Kinokuniya, Tokyo, pp. 749-770 (1987)

[34] Viehweg, E.: Canonical divisors and the additivity of the Kodaira dimension for morphisms of relative dimension one. Compos. Math. 35, 197-223 (1977)

[35] Yamaki, K.: Cornalba-Harris equality for semistable hyperelliptic curves in positive characteristic. Asian J. Math. 8, 409-426 (2004)

[36] Zhang, S.-W.: Gross-Schoen cycles, dualising sheaves, and triple product $L$-series. Preprint 